\title{
Evaluating the Effect of Tissue Anisotropy on Brain Tumor Growth using a Mechanically-coupled Reaction-Diffusion Model
}

\author{
Daniel Abler ${ }^{\star, \dagger}$, Russell C. Rockne ${ }^{\dagger}$, Philippe Büchler ${ }^{\star}$ \\ ${ }^{\star}$ Institute for Surgical Technology and Biomechanics \\ University of Bern, Bern, Switzerland \\ daniel.abler@istb.unibe.ch, philippe.buechler@istb.unibe.ch \\ ${ }^{\dagger}$ Beckman Research Institute \\ City of Hope, Duarte, CA, USA \\ dabler@coh.org,rrockne@coh.org
}

Keywords: glioma, anisotropy, DTI, mass effect, reaction diffusion model, biomechanics.

\begin{abstract}
Glioblastoma (GBM), the most frequent malignant brain tumor in adults, is characterized by rapid growth and healthy tissue invasion. Long-term prognosis for GBM remains poor with median overall survival between 1 y to 2 y [15]. GBM presents with different growth phenotypes, ranging from invasive tumors without notable mass-effect to strongly displacing lesions. Biomechanical forces, such as those resulting from displacive tumor growth, shape the tumor environment and contribute to tumor progression [9].

We present an extended version of a mechanically-coupled reaction-diffusion model of brain tumor growth [1] that simulates tumor evolution over time and across different brain regions using literature-based parameter estimates for tumor cell proliferation, as well as isotropic motility, and mechanical tissue properties. This model yielded realistic estimates of the mechanical impact of a growing tumor on intra-cranial pressure. However, comparison to imaging data showed that asymmetric shapes could not be reproduced by isotropic growth assumptions.

We modified this model to account for structural tissue anisotropy which is known to affect the directionality of tumor cell migration and may influence the mechanical behavior of brain tissue. Tumors were seeded at multiple locations in a human MR-DTI brain atlas and their spatio-temporal evolution was simulated using the Finite-Element Method. We evaluated the impact of tissue anisotropy on the model's ability to reproduce the aspherical shapes of real pathologies by comparing predicted lesions to publicly available GBM imaging data.

We found the impact on tumor shape to be strongly location dependent and highest for tumors located in brain regions that are characterized by a single dominant white matter direction, such as the corpus callosum. However, despite strongly anisotropic growth assumptions, all simulated tumors remained more spherical than real lesions at the corresponding location and similar volume. This finding is in agreement with previous studies [17, 6] suggesting that anisotropic cell migration along white matter fiber tracks is not a major determinant of tumor shape in the setting of reaction-diffusion based tumor growth models and for most locations across the brain.
\end{abstract}




\section{INTRODUCTION}

Gliomas are the most frequent primary brain tumors in adults (70\%) [15]. Glioblastoma multiformae (GBM) is the most malignant sub-type of glioma, accounting for about $50 \%$ of diffuse gliomas. GBM infiltrates surrounding healthy tissue, grows rapidly and forms a necrotic core of high cell density which is frequently accompanied by compression and displacement of surrounding tissue. Despite aggressive treatment, long-term prognosis remains poor with median overall-survival below 1.5 years [15].

Invasive growth and mass effect are the macroscopic hallmarks of GBM. Variability can be observed with regard to these characteristics, ranging from predominantly invasive tumors without notable mass-effect to strongly displacing ones that induce higher mechanical stresses and result in healthy tissue deformation, midline shift or herniation. These solid stresses play an important role for tumor evolution [9], which suggests that biomechanical factors have direct implications not only on the biophysical level, but may affect treatment response and outcome.

We have previously developed a mechanically-coupled reaction-diffusion model of brain tumor growth that accounts for tumor mass-effect [1]. The framework simulates tumor evolution over time and across different brain regions using literature-based parameter estimates for tumor cell proliferation, as well as isotropic motility, and mechanical tissue properties. This model yielded realistic estimates of the mechanical impact of a growing tumor on intra-cranial pressure. However, comparison to imaging data showed that asymmetric shapes could not be reproduced by isotropic growth assumptions.

To investigate the role of tissue anisotropy on simulated tumor shape, we extended our simulation framework to take into account tissue structure. White matter consists predominantly of aligned axonal fibers. Their orientation can be inferred from Magnetic-resonance (MR) diffusion tensor imaging (DTI) which measures water diffusion along different directions in space. As diffusion is constrained transverse to fiber direction, MR-DTI provides structural information of brain tissue. Information from MR-DTI has previously been used to inform tumor cell migration behavior in mathematical models of brain tumor growth, see [17, table 1] for an overview of previous work.

The few studies that have investigated the effect of tissue anisotropy on larger patient cohorts found it to have a beneficial, but relatively small effect on their models' ability to reproduce real tumor shapes. Employing the anisotropic glioma spread model of [14], [17] investigated the effect of tissue anisotropy without mass-effect. Their study on 10 cases showed an improved ability to approximate tumor shapes (average increase in Jaccard score by $0.03 \pm 0.03$, about $5 \%$ relative to the isotropic case) when including patient-specific DTI information and personalized estimates for a patient-specific anisotropy parameter that describes the sensitivity of cancer cells to the underlying brain structure. Only few studies [5, 3, 6] took into account the tumor's mass effect when investigating the effect of tissue anisotropy. Simulation results of 9 low-grade glioma cases were reported by [6], using patient-specific DTI information, nonpersonalized growth parameters and an isotropic visco-elastic material model for brain tissue. Availability of DTI information in their study improved Jaccard Score (Dice Index) between simulated and actual tumor by $\leq 2.40 \%(\leq 1.50 \%)$.

In this study, we investigate the combined effect of anisotropic growth and mechanical 


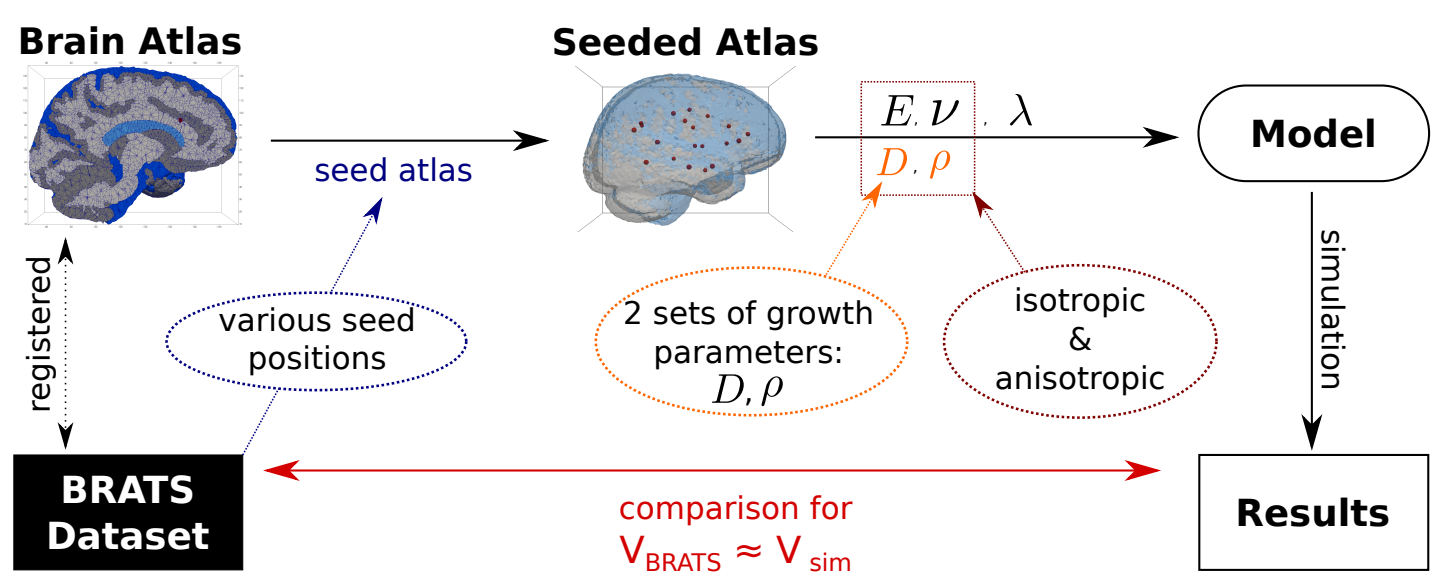

Figure 1: Tumor growth evolution was simulated in a healthy brain atlas for two sets of growth parameters $(D, \rho)$, and isotropic and anisotropic tissue properties. Simulated tumors were compared to subjects from the BRATS data set at approximately identical volume.

characteristics on tumor shape in a mechanically-coupled reaction-diffusion model of invasive glioma growth by comparing simulation results obtained from isotropic and anisotropic material assumptions.

\section{MATERIALS \& METHODS}

Figure 1 illustrates the study setup: Virtual tumors were seeded in an atlas of healthy brain anatomy at representative locations extracted from 10 subjects of the BRATS $2013^{1}$ [12, 11] training dataset. Figure 2 shows the spatial distribution of the selected lesions in a human brain atlas. Tumor growth evolution was simulated for isotropic and anisotropic tissue properties and two sets of growth parameter choices, corresponding to diffuse and nodular growth characteristics, respectively. Virtually grown and real tumors were compared when the simulated tumor volume equaled the tumor volume of the corresponding subjects from the BRATS dataset.

\subsection{Mathematical Model}

The mathematical model used in this study captures three interrelated aspects of macroscopic glioma growth [1]: Cell proliferation, invasion of tumor cells into the surrounding healthy tissue, and tissue deformation due to the tumor-induced mass-effect.

We model the invasive growth of glioma phenomenologically as as a Reaction-Diffusion (RD) process [19], representing cell migration by passive diffusion:

$$
\frac{\partial q}{\partial t}=\nabla \cdot(\hat{\boldsymbol{D}} \nabla q)+\rho q(1-q)
$$

with normalized cancer cell concentration $q(\boldsymbol{r}, t)$ and diffusion tensor $\hat{\boldsymbol{D}}=\hat{\boldsymbol{D}}(\boldsymbol{r})$. Tumor growth is modeled as logistic growth process with proliferation rate $\rho$.

Similarly to [5, 3], the tissue-displacing mass-effect of the growing tumor is represented phenomenologically using a linear-elastic solid mechanics approach. It relies on the assumption

\footnotetext{
${ }^{1}$ https : //www . smir.ch/BRATS/Start2013
} 

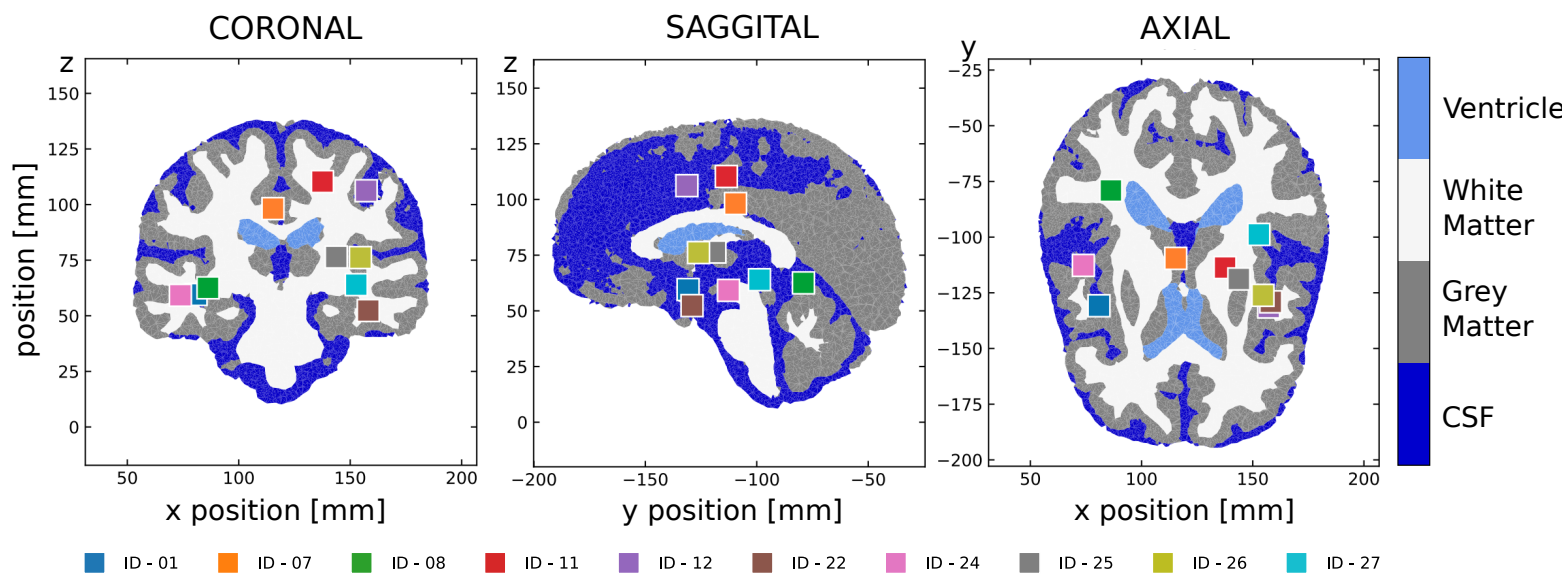

Figure 2: Tumor center-of-mass positions of 10 selected BRATS cases projected onto central planes of SRI24 atlas.

that the creation of new tumor cells leads to volumetric increase of the tumor and thus results in an expansion of the affected brain tissue. The volumetric increase is modeled by introducing a growth-induced strain component $\hat{\boldsymbol{\epsilon}}^{\text {growth }}(q)$, so that

$$
\hat{\boldsymbol{\epsilon}}^{\text {total }}(\boldsymbol{u}, q)=\hat{\boldsymbol{\epsilon}}^{\text {elastic }}(\boldsymbol{u})+\hat{\boldsymbol{\epsilon}}^{\text {growth }}(q) .
$$

where displacements $\boldsymbol{u}$ are obtained from solving the linear-momentum equilibrium equation with stress $\hat{\boldsymbol{\sigma}}(\boldsymbol{u})$ and strain $\hat{\boldsymbol{\epsilon}}^{\text {total }}(\boldsymbol{u})$ linked by a linear constitutive relationship.

Additionally, we assume a linear coupling between tumor cell concentration and growthinduced strain

$$
\hat{\boldsymbol{\epsilon}}^{\text {growth }}(q)=\hat{\boldsymbol{\lambda}} q=\lambda \mathbb{1} q,
$$

with isotropic coupling strength $\lambda$.

\subsection{Simulation Domain}

We used the SRI24 ${ }^{2}$ [16] atlas of normal human brain anatomy to define the simulation geometry with tissue classes. Release 2.0 of the atlas provides separate tissue labels for White Matter (WM), Grey Matter (GM) and Cerebrospinal Fluid (CSF). We divided the CSF domain into two compartments to distinguish fluid-filled brain ventricles from the remaining CSF, surrounding the brain tissue. Additionally, we extracted the map of dominant Diffusion-Tensor Imaging (DTI) eigenvectors from an initial release (v0.0) of the atlas. This information was interpreted as local dominant orientation of axon fibers and was used to inform diffusion and mechanical tissue parameters in the anisotropic simulation scenario. Finally, all relevant components of the atlas were registered to fit the spatial orientation of the BRATS datasets.

The tumor center-of-mass position was computed for each of the 10 selected subjects, based on the tumor volume visible on T1-weighted contrast-enhanced (T1c) MR imaging ${ }^{3}$. For each

\footnotetext{
${ }^{2}$ https://www.nitrc.org/projects/sri24/

${ }^{3}$ We identified the T1c volume with the combined segmentation labels for necrotic, enhanced, non-enhanced tumor.
} 

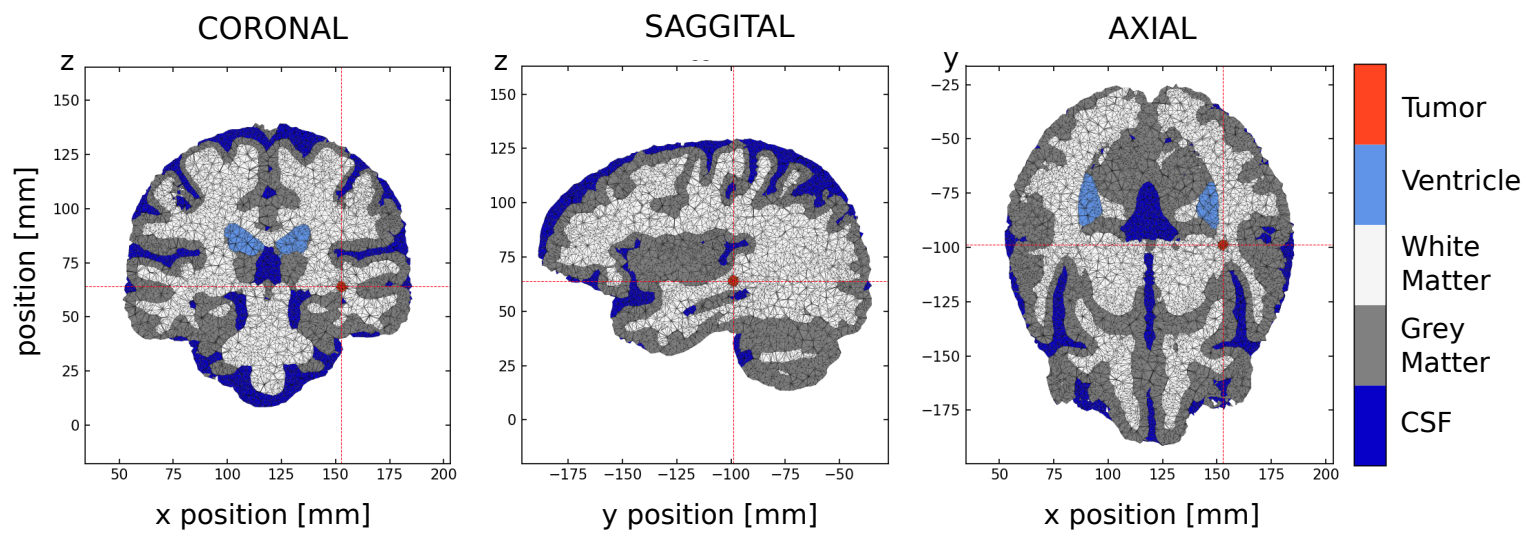

Figure 3: Projections through seeded SRI24 atlas. An exemplary seed location is shown in the tetrahedral mesh used for simulation.

subject, a spherical tumor seed ( $2 \mathrm{~mm}$ radius) was introduced in the atlas label map at the corresponding center-of-mass position, and a tetrahedral mesh was generated (approximately 320000 elements) using $\mathrm{CGAL}^{4}$ and $\mathrm{VTK}^{5}$ libraries. DTI information from the SRI24 atlas was then interpolated over the seeded mesh. Figure 3 shows coronal, saggital and axial views through an exemplary seeded and meshed simulation domain.

\subsection{Simulation Assumptions}

To compare the effect of tissue anisotropy on the simulation of tumor characteristics, two different simulation scenarios were considered, assuming isotropic and anisotropic material properties, respectively.

In both cases, the brain tissues WM and GM were modeled as linear-elastic materials. The CSF of the brain ventricles was modeled as compressible to account for physiological mechanisms that compensate elevated intra-cranial pressure [21], whereas the remaining CSF was modeled as nearly incompressible. Simulations were run with two distinct growth parameter sets corresponding to nodular and diffuse growth characteristics with $\rho / D \geq 1.35 \mathrm{~mm}^{-2}$ and $\rho / D \leq 0.37 \mathrm{~mm}^{-2}$ [2], respectively. A maximum tumor-induced strain of $15 \%$ [13] was assumed, $\lambda=0.15$, and an initial condition of $q=1$ over the entire volume of the tumor seed was imposed. Deformation of the brain surface and escape of tumor cells from the brain was constrained by zero-displacement and zero-flux boundary conditions at surface nodes. The mathematical model was solved using the Finite Element Method. It was implemented in Abaqus (Simulia, Dassault Systémes) as fully-coupled thermal stress analysis using 4-node linear elements (C3D4T) with the tumor mass-effect being represented by volumetric thermal expansion.

Isotropic Scenario In the isotropic simulation scenario, diffusion and mechanical tissue behavior were assumed isotropic using the parameter values summarized in table 1 and table 2 for

\footnotetext{
${ }^{4}$ https: //www.cgal.org

${ }^{5}$ https://www.vtk.org
} 


\begin{tabular}{lcccccc}
\hline growth type & $\begin{array}{c}\rho \\
{[1 / \mathrm{d}]}\end{array}$ & $\begin{array}{c}D_{\text {avg }} \\
{\left[\mathrm{mm}^{2} / \mathrm{d}\right]}\end{array}$ & $\begin{array}{c}D_{\mathrm{avg}} / \rho \\
{\left[\mathrm{mm}^{2}\right]}\end{array}$ & $\begin{array}{c}\rho / D_{\text {avg }} \\
{\left[\mathrm{mm}^{-2}\right]}\end{array}$ & $\begin{array}{c}D_{\mathrm{GM}} \\
{\left[\mathrm{mm}^{2} / \mathrm{d}\right]}\end{array}$ & $\begin{array}{c}D_{\mathrm{WM}} \\
{\left[\mathrm{mm}^{2} / \mathrm{d}\right]}\end{array}$ \\
\hline nodular & 0.082 & 0.053 & 0.650 & 1.540 & 0.020 & 0.101 \\
diffuse & 0.037 & 0.105 & 2.855 & 0.350 & 0.040 & 0.200 \\
\hline
\end{tabular}

Table 1: Reaction-Diffusion parameter sets $(D, \rho)$, representing nodular and diffuse growth. Tissue-specific motility estimates $\left(D_{\mathrm{WM}}, D_{\mathrm{GM}}\right)$ are based on the assumption that $D_{\text {avg }}$ was measured in a tissue volume containing equal portions of GM and WM, and $D_{\mathrm{WM}}=5 D_{\mathrm{GM}}[19]$.

\begin{tabular}{lcc}
\hline Tissue & $E$ & $\nu$ \\
& {$[\mathrm{kPa}]$} & \\
\hline W/G Matter & 3.00 & 0.45 \\
Tumor & 6.00 & 0.45 \\
CSF (Ventricles) & 1.00 & 0.30 \\
CSF (other) & 1.00 & 0.49 \\
\hline
\end{tabular}

Table 2: Mechanical tissue properties (isotropic case), informed by [21].

the considered tissue types. The linear material model was fully characterized by two parameters, Poisson ratio $\nu$ and Young's modulus $E$. Values for the Young's modulus of brain tissue and tumor were adopted from [21].

Anisotropic Scenario In the anisotropic simulation scenario, white matter fiber directionality was taken into account and the tissue was modeled as transversely isotropic material with different material properties along $(\|)$ and orthogonal to $(\perp)$ the fibers.

Tumor cell motility along fiber direction (WM) was assumed identical to the isotropic case $D_{\mathrm{W}}^{\|}=D_{\mathrm{W}}^{\text {iso }}$, whereas a significantly lower motility was used in the transverse direction $D_{\mathrm{W}}^{\perp}=0.01 D_{\mathrm{W}}^{\text {iso }}$. Due to reduced fiber alignment, cell motility in grey matter was modeled as isotropic $[5,3]$ with the value indicated in table 1 . We chose a very high ratio $D_{\mathrm{W}}^{\|} / D_{\mathrm{W}}^{\perp}=100$ to investigate the effect of growth anisotropy. For comparison, [6] assumed a ratio of 5; [10] varied this ratio between 5 to 100 and found the best "de visu" fit for a ratio of 10 .

Linear-elastic mechanical tissue properties of the transversely isotropic situation can be expressed in terms of seven engineering constants: Two Young's moduli that describe the stresses resulting from uniaxial stretch parallel $E^{\|}$and perpendicular $E^{\perp}$ to the fiber axis. Two shear moduli that describe shear stresses in planes parallel to $\left(\mu^{\|}\right)$and normal to $\left(\mu^{\perp}\right)$ the fiber axis. Three Poisson ratios $\nu^{\| \perp}, \nu^{\perp \|}, \nu^{\perp \perp}$ that describe the strain in one direction (\| or $\perp$ ) that arises from stretch in another orthogonal direction ( $\|$ or $\perp$ ). Only five of these seven parameters are 


\begin{tabular}{lccccccc}
\hline Tissue & $\begin{array}{c}E^{\|} \\
{[\mathrm{kPa}]}\end{array}$ & $\begin{array}{c}E^{\perp} \\
{[\mathrm{kPa}]}\end{array}$ & $\begin{array}{c}\mu^{\|} \\
{[\mathrm{kPa}]}\end{array}$ & $\begin{array}{c}\mu^{\perp} \\
{[\mathrm{kPa}]}\end{array}$ & $\nu^{\| \perp}$ & $\nu^{\perp \|}$ & $\nu^{\perp \perp}$ \\
\hline White Matter & 4.50 & 1.50 & 0.56 & 0.40 & 0.45 & 0.15 & 0.85 \\
Tumor (if in WM) & 9.00 & 3.00 & 1.12 & 0.80 & 0.45 & 0.15 & 0.85 \\
\hline
\end{tabular}

Table 3: Mechanical tissue properties (anisotropic simulation scenario), assuming transverse symmetry with directions along $(\|)$ and orthogonal to $(\perp)$ fiber direction. Material properties for GM and CSF were those from table 2

typically independent since additionally:

$$
\begin{aligned}
\frac{\nu^{\| \perp}}{E^{\|}} & =\frac{\nu^{\perp \|}}{E^{\perp}} \\
\mu^{\perp} & =\frac{E^{\perp}}{2\left(1+\nu^{\perp \perp}\right)} .
\end{aligned}
$$

To estimate parameters of that model, we assume a fiber reinforcement effect in white matter that increases resistance against stretch along the fiber direction, $E_{W}^{\|}=3 \cdot E_{W}^{\perp}$, from observations on lamb corpus callosum $E^{\|} / E^{\perp} \approx 6.5$ [7] and porcine corona radiata $E^{\|} / E^{\perp} \approx 2.7$ [20, 7]. Based on the material parameters used for the isotropic cases, we defined the Young's moduli of white matter so that $E_{\mathrm{WM}}^{\|}>E_{\mathrm{GM}}^{\text {iso }}>E_{\mathrm{WM}}^{\perp}$. We assume $\nu^{\| \perp}=\nu^{\text {iso }}$, so that $\nu^{\perp \|}$ follows from eq. (4a) and $\nu^{\perp \perp}=1-\nu^{\perp \|}$. This allows us to compute $\mu^{\perp}$ from eq. (4b). We then compute $\mu^{\|}=1.4 \mu^{\perp}$ [7]. Resulting mechanical model parameters for white matter are summarized in table 3 .

\subsection{Analysis}

Two different tumor detection thresholds were used to evaluate simulation results: $c_{\mathrm{T} 1 \mathrm{c}}=$ 0.80 and $c_{\mathrm{T} 2}=0.16$ corresponding to tumor features visible on T1-weighted contrast enhanced (T1c) and T2-weighted (T2) MRI imaging [18], respectively. Simulations were run until the simulated tumor had reached the T1c volume of the corresponding BRATS subject. Tumors corresponding to $\mathrm{T} 1 \mathrm{c}$ and $\mathrm{T} 2$ visibility threshold were extracted at multiples of $5 \mathrm{~mm}$ increments in equivalent radius computed from the simulated T1c volume. For each tumor volume, the following measures were computed: a) Tumor aspect ratio, as the ratio between shortest and longest axis of the smallest bounding box around the tumor. A value of 1 corresponds to a spherical tumor shape; values closer to 0 indicate aspherical (elongated, oblate or asymmetric) shapes. b) Tumor nodularity, as the ratio of T1c and T2 tumor volumes. A value close to 1 corresponds to a very well delineated, nodal tumor, whereas values closer to 0 indicate diffuse growth.

The same measures were computed from BRATS segmentations by identifying the T1c tumor volume with labels \{necrotic, non-enhancing tumor, enhancing tumor\} and the T2 volume with labels \{necrotic, non-enhancing tumor, enhancing tumor, edema\}. Measures derived from simulated tumors and real pathologies were compared at similar volumes $V_{\mathrm{T} 1 \text { c, sim }} \approx V_{\mathrm{T} 1 \text {, BRATS }}$. 


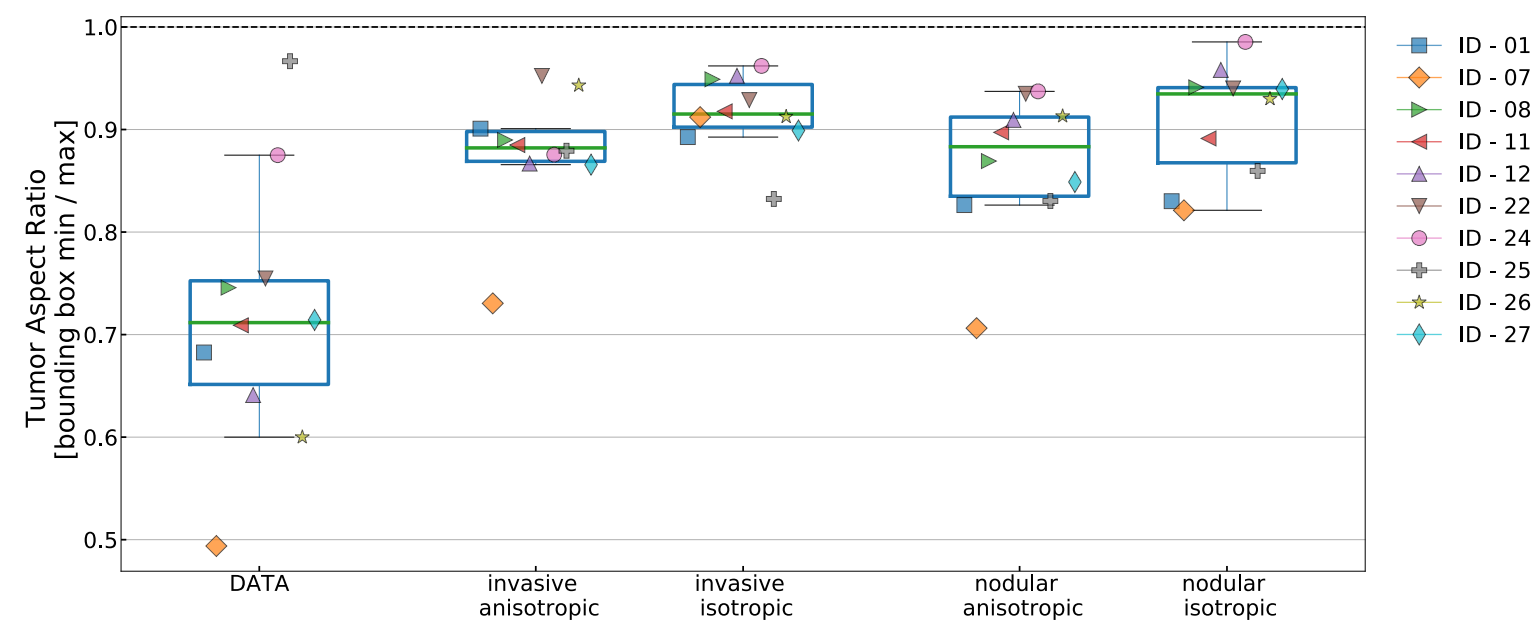

Figure 4: Aspect ratio of BRATS T1c lesions and simulated tumors for diffuse/nodular growth parameterization and isotropic/anisotropic tissue properties. A value of 1 indicates a spherical shape, whereas lower values correspond to oblate or elongated shapes.

\section{RESULTS}

Tumor growth evolution and tissue deformation were simulated for all 10 selected BRATS subjects, growth parameterizations (nodular, diffuse) and tissue structure scenarios (isotropic, anisotropic).

The anisotropic growth scenario showed an average $4.3 \pm 6.2 \%$ reduction of tumor aspect ratio compared to isotropic growth assumptions. Similar average impact on tumor shape was found for diffuse $(3.9 \pm 7.6 \%)$ and nodular $(4.7 \pm 4.6 \%)$ growth parameterizations. However, both isotropic and anisotropic growth assumptions resulted in simulated tumor shapes that were more spherical than the corresponding BRATS lesions, fig. 4.

The effect of tissue anisotropy on simulated tumor shape was strongly dependent on seed location: Tumors grown from seeds located deep in WM (ID-07, ID-27) and adjacent to the lateral ventricle (ID-08) exhibited a strong effect of tissue anisotropy. Seeds located closer to WM/GM interfaces (ID-11, ID-12, ID-22, ID-24) showed mixed effects, while those located in GM (ID-01, ID-25, ID-26) experienced only small effects, fig. 5. These observations are consistent with our chosen parameterization approach that considers GM to be isotropic. The effect of tissue anisotropy on shape was particularly pronounced for ID-07 located medially in the corpus callosum, a region of highly aligned nerve fibers.

Tumor nodularity extracted from BRATS images (DATA in Figure 6) differed across the selected cases. For each simulated BRATS case, the computed nodularity measure was consistent with growth parameterization: lower for diffuse and higher for nodal growth. In most cases the anisotropic growth scenario resulted in more nodular tumors compared to isotropic growth assumptions, due to reduced overall diffusivity. Despite identical growth parameterization (nodular, diffuse), the computed nodularity of simulated tumors differed across BRATS subjects. This effect can be attributed to differences in the growth environment (WM, GM, boundary, constrained by $\mathrm{CSF} /$ ventricle) resulting in distinct average growth parameters for 


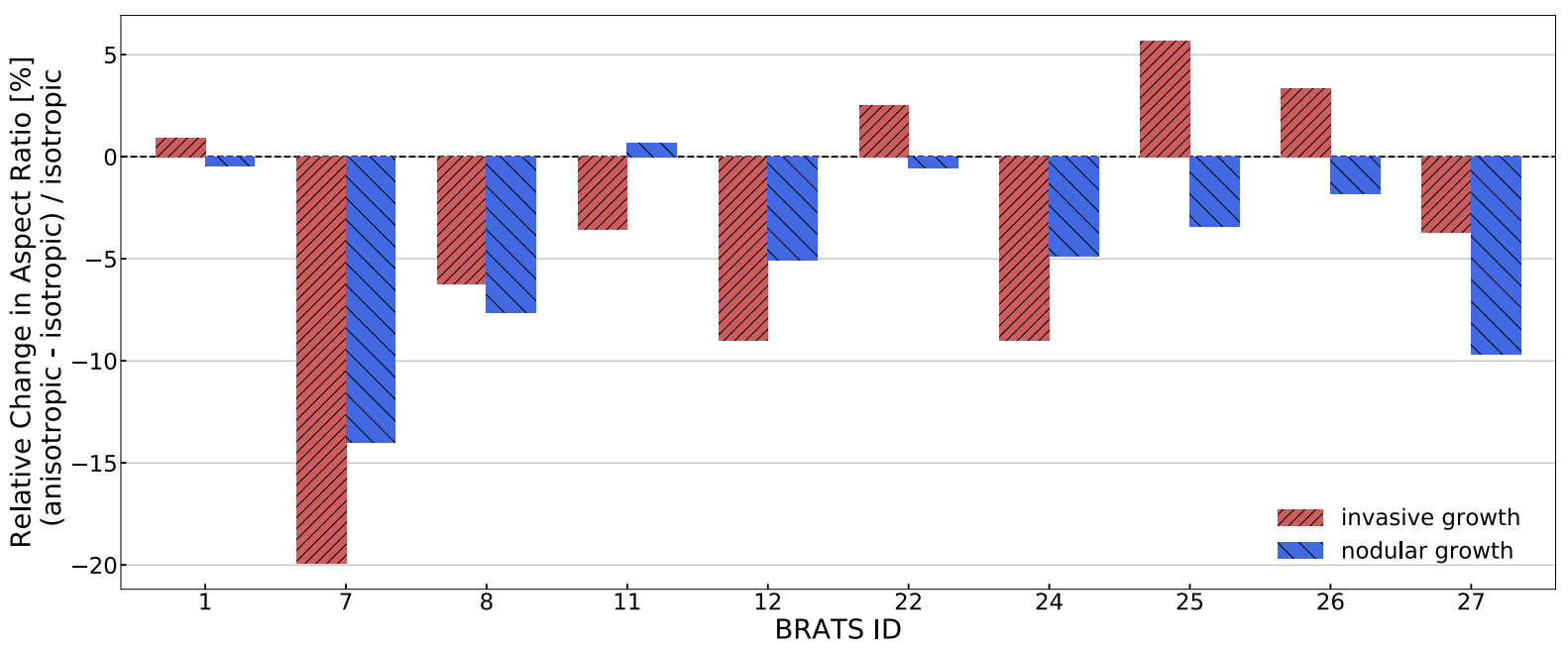

Figure 5: Relative change in tumor aspect ratio between isotropic and anisotropic configurations. A negative value corresponds to a decrease in aspect ratio due to anisotropic material properties.

each lesion.

\section{DISCUSSION}

This study explored the effect of tissue anisotropy, affecting tumor cell migration and mechanical properties, on glioma growth simulations in a 3D brain atlas. In agreement with our model parameterization, we found tissue anisotropy to result in reduced tumor shape symmetry for tumors located in WM and for some lesions at the WM/GM interface. However, despite a strongly anisotropic choice of diffusion parameters, $D_{\mathrm{W}}^{\|} / D_{\mathrm{W}}^{\perp}=100$, all simulated tumors remained more spherical than real lesions at the corresponding location and similar volume.

Our findings confirm previous simulation studies $[17,6]$ suggesting that anisotropic cell migration along WM fiber tracks is not a major determinant of tumor shape in the setting of reaction-diffusion based tumor growth models and for most locations across the brain. Exceptions might apply for tumors located in brain regions where a single dominant fiber direction prevails over multiple contiguous centimeters. For example, in this study we observed the highest relative change in aspect ratio due to tissue anisotropy, $14 \%$ to $20 \%$, for a medially located GBM in the corpus callosum (ID-07).

Large variability in tumor nodularity for identical growth parameterizations (diffuse/nodular) across different brain locations, Figure 6, indicates that 3D tumor growth is strongly affected by the tissue composition of a tumors' growth domain. We hypothesize that the interplay between tissue composition, spatial constraints and resulting mechanical forces may exceed the effect of tissue anisotropy on tumor growth, possibly giving rise to location-specific growth archetypes of GBM.

While our model computed tumor mass-effect and resulting healthy tissue deformation, neither this nor similar previous modeling studies for human GBM [17, 6] captured the growthinhibiting effect of solid stress [8]. The present study has further limitations: $a$ ) We seeded a 


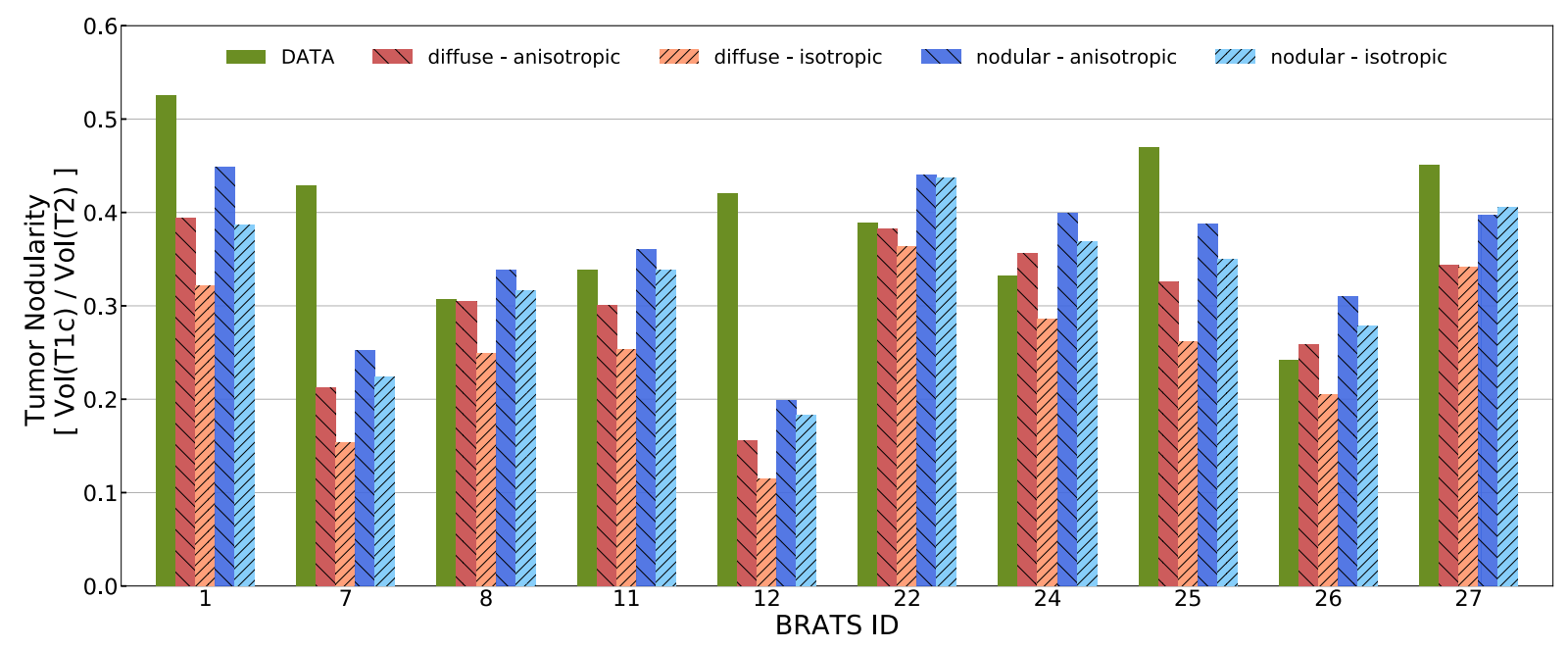

Figure 6: Tumor nodularity of BRATS lesions and simulated tumors for diffuse / nodular growth parameterization and isotropic / anisotropic tissue properties. A value close to 1 corresponds to a nodular tumor, whereas smaller values indicate diffuse growth.

brain atlas at the center-of-mass position of actual lesions. A mismatch between patient and atlas anatomy and/or asymmetric growth may have resulted in the simulated tumor growth process starting in a different brain tissue, which could significantly affect the tumor's simulated evolution. This may explain shape discrepancies for some of the BRATS cases, such as ID-25 (ID-26) which has a very high (low) aspect ratio in the BRATS dataset, but ranges among the simulated tumor shapes with lowest (higher) aspect ratio. $b$ ) DTI information was derived from an atlas of the healthy human brain so that possible changes in local tissue structure due to tumor growth were not taken into account. We considered brain tissues to be either isotropic (GM) or anisotropic (WM), not distinguishing varying degrees of anisotropy within each tissue class. Also, possible differences in patient-specific sensitivity of cancer cells to the underlying brain structure were not taken into account. c) This study relied on a linear elastic material model with estimates for mechanical tissue anisotropy derived from evidence of animal brain tissue characterization. Recent evidence [4] suggests that a Ogden material model best captures the mechanical response of human brain tissue and questions its direction dependence.

This study investigated the joint impact of tumor mass effect and tissue anisotropy on simulated tumor shape. Further work is needed to combine the individual contributions of structural anisotropy, tissue composition and mechanical growth constraints in a way to best reproduce GBM growth characteristics. We will address the limitations of this study in future work that aims to investigate the determinants of glioma growth for typical tumor locations.

\section{Acknowledgements}

The research leading to these results has received funding from the European Union's Horizon 2020 research and innovation programme under the Marie Skłodowska-Curie grant agreement No 753878. Calculations were performed on UBELIX (http://www.id.unibe.ch/ hpc), the HPC cluster at the University of Bern. 


\section{References}

[1] D. Abler et al. "Evaluation of a Mechanically Coupled Reaction-Diffusion Model for Macroscopic Brain Tumor Growth". In: Computer Methods in Biomechanics and Biomedical Engineering. Ed. by A. Gefen et al. Cham: Springer International Publishing, 2018, pp. 57-64.

[2] A. L. Baldock et al. "Patient-Specific Metrics of Invasiveness Reveal Significant Prognostic Benefit of Resection in a Predictable Subset of Gliomas". In: PLoS ONE 9.10 (Oct. 2014), e99057.

[3] P.-Y. Bondiau et al. "Biocomputing: Numerical Simulation of Glioblastoma Growth Using Diffusion Tensor Imaging”. In: Physics in Medicine and Biology 53.4 (Feb. 2008), pp. 879-893.

[4] S. Budday et al. "Mechanical Characterization of Human Brain Tissue". In: Acta Biomaterialia 48 (Jan. 2017), pp. 319-340.

[5] O. Clatz et al. "Realistic Simulation of the 3-D Growth of Brain Tumors in MR Images Coupling Diffusion with Biomechanical Deformation". In: IEEE Transactions on Medical Imaging 24.10 (Oct. 2005), pp. 1334-1346.

[6] A. Elazab et al. "Post-Surgery Glioma Growth Modeling from Magnetic Resonance Images for Patients with Treatment”. In: Scientific Reports 7.1 (Dec. 2017).

[7] Y. Feng et al. "Measurements of Mechanical Anisotropy in Brain Tissue and Implications for Transversely Isotropic Material Models of White Matter". In: Journal of the Mechanical Behavior of Biomedical Materials 23 (July 2013), pp. 117-132.

[8] G. Helmlinger et al. "Solid Stress Inhibits the Growth of Multicellular Tumor Spheroids". In: Nature Biotechnology 15.8 (Aug. 1997), pp. 778-783.

[9] R. K. Jain et al. "The Role of Mechanical Forces in Tumor Growth and Therapy". In: Annual Review of Biomedical Engineering 16.1 (July 2014), pp. 321-346.

[10] S. Jbabdi et al. "Simulation of Anisotropic Growth of Low-Grade Gliomas Using Diffusion Tensor Imaging”. In: Magnetic Resonance in Medicine 54.3 (2005), pp. 616-624.

[11] M. Kistler et al. "The Virtual Skeleton Database: An Open Access Repository for Biomedical Research and Collaboration". In: J Med Internet Res 15.11 (Nov. 2013), e245.

[12] B. Menze et al. "The Multimodal Brain Tumor Image Segmentation Benchmark (BRATS)". In: IEEE Transactions on Medical Imaging (2014), p. 33.

[13] A. Mohamed et al. "Finite Element Modeling of Brain Tumor Mass-Effect from 3D Medical Images". In: Medical Image Computing and Computer-Assisted Intervention-MICCAI 2005. Lecture Notes in Computer Science 3749. Springer, 2005, pp. 400-408.

[14] K. Painter et al. "Mathematical Modelling of Glioma Growth: The Use of Diffusion Tensor Imaging (DTI) Data to Predict the Anisotropic Pathways of Cancer Invasion". In: Journal of Theoretical Biology 323 (Apr. 2013), pp. 25-39. 
[15] D. Ricard et al. "Primary Brain Tumours in Adults". In: The Lancet 379.9830 (May 2012), pp. 1984-1996.

[16] T. Rohlfing et al. "The SRI24 Multichannel Atlas of Normal Adult Human Brain Structure”. In: Human Brain Mapping 31.5 (Dec. 2009), pp. 798-819.

[17] A. Swan et al. "A Patient-Specific Anisotropic Diffusion Model for Brain Tumour Spread". In: Bulletin of Mathematical Biology (May 2017).

[18] K. R. Swanson et al. "A Mathematical Modelling Tool for Predicting Survival of Individual Patients Following Resection of Glioblastoma: A Proof of Principle". In: British Journal of Cancer 98.1 (Jan. 2008), pp. 113-119.

[19] K. R. Swanson et al. "A Quantitative Model for Differential Motility of Gliomas in Grey and White Matter”. In: Cell Proliferation 33.5 (Oct. 2000), pp. 317-329.

[20] F. Velardi et al. "Anisotropic Constitutive Equations and Experimental Tensile Behavior of Brain Tissue". In: Biomechanics and Modeling in Mechanobiology 5.1 (Mar. 2006), pp. 53-61.

[21] A. Wittek et al. "Patient-Specific Non-Linear Finite Element Modelling for Predicting Soft Organ Deformation in Real-Time; Application to Non-Rigid Neuroimage Registration". In: Progress in Biophysics and Molecular Biology. Special Issue on Biomechanical Modelling of Soft Tissue Motion 103.2-3 (Dec. 2010), pp. 292-303. 